\title{
GONVOLUTION TRANSFORMS RELATED TO NON-HARMONIC FOURIER SERIES
}

\author{
D. B. SUMNER
}

1. Introduction. Widder has pointed out (2, p. 219) in connection with Wiener's fundamental work on the operational calculus (1, pp. 557-584), that the convolution transform

$$
f(x)=\int_{-\infty}^{\infty} G(x-t) \phi(t) d t
$$

will be inverted by the operator $D E(D)$, where $D=d / d x$, and

$$
1 / w E(w)=\int_{-\infty}^{\infty} \exp (-x w) G(x) d x,
$$

where a suitable interpretation must be found for $E(D)$. Cases where $E(w)$ is entire have been considered by Widder (2, pp. 217-249; 3, pp. 7-60), Hirschman and Widder (4, pp. 659-696; 8, pp. 135-201), and the author (5).

The most general method of interpreting $E(D)$ is as $\lim _{n \rightarrow \infty} P_{n}(D)$, where $P_{n}(w)$ is a polynomial of degree $n$, the method requiring a knowledge of $f(x)$ only for real values of its argument. However in cases where more is known about $E(w)(4$, p. $692 ; 5$, pp. $174-183 ; 6$, p. 219$)$, it is possible to represent $E(w)$ as an integral, when the computations are simpler, but it is necessary to have $f(x)$ defined for complex arguments.

The purpose of this article is to consider convolution transforms for which the invertor function $E(w)$ is entire, is not necessarily even, and can be represented by a Fourier-Lebesgue integral. The real numbers which are taken to be the zeros of $E(w)$ are a generalization of the non-harmonic Fourier exponents discussed by Levinson (7, pp. 47-57). The classical Stieltjes transform (3), and the generalized form of it (5), are particular cases. The assumptions made about the zeros of $E(w)$ are sufficient to establish all properties needed, and no integrability condition is postulated for $E(u)$.

2. Definitions. We suppose throughout that

$$
\begin{gathered}
\lambda_{n}=\rho+n-\delta+2 \delta \alpha_{n}, \mu_{n}=n-\delta+2 \delta \beta_{n} \quad(n=1,2, \ldots), \\
0 \leqslant \alpha_{n}, \beta_{n} \leqslant 1, \quad 0 \leqslant \delta<\frac{1}{4}, \quad 0 \leqslant \rho<1-2 \delta ; \\
E(w)=\prod_{1}^{\infty}\left(1-w / \lambda_{n}\right)\left(1+w / \mu_{n}\right) ; \\
G(z)=\lim _{R \rightarrow \infty}(2 \pi i)^{-1} \int_{c-i R}^{c+i R} \exp (z w) d w / w E(w), \quad 0<c<\lambda_{1} .
\end{gathered}
$$

The symbols $A, A_{k}$ denote absolute constants throughout.

Received September 12, 1952; in revised form July 15, 1954. 
3. Some properties of $E(w)$. The numbers $\lambda_{n}, \mu_{n}$ are those used by Levinson (7, pp. 47-57) in his work on non-harmonic Fourier series. With the notation $w=u+i v=r \exp (i \phi)$, Levinson's methods may be used to establish the following inequalities:

$$
\begin{aligned}
|E(\mathrm{w})| & \leqslant A \exp (\pi|v|) / \mathrm{r}^{\rho+1-4 \delta}, \\
|E(w)| & \geqslant A \exp (\pi|v|) / r^{\rho+1-4 \delta}, \text { provided that } \\
r \geqslant 1, \quad\left|w-r_{n}\right| & \geqslant \Delta>0, \quad r_{n}=\lambda_{n} \text { or }-\mu_{n} ; \\
\left|E^{\prime}\left(r_{n}\right)\right|^{-1} & \leqslant A r^{\rho+1+4 \delta}
\end{aligned}
$$

(3.4) there exists a constant $q, 1<q \leqslant 2$, such that $E(u) \in L^{q}(-\infty, \infty)$.

For the behaviour of $E(w)$ along the imaginary axis, we establish the more precise inequalities

$$
\begin{gathered}
|E(i v)| \leqslant A \exp (\pi|v|) /|v|^{\rho+1-2 \delta},|E(i v)| \geqslant A \exp (\pi|v|) /|v|^{\rho+1+2 \delta} \\
|\operatorname{amp} E(i v \theta) / E(i v)|<A(1-\theta),
\end{gathered}
$$

where $0<\theta \leqslant 1$, and the constant is independent of $v$;

$$
|E(i v \theta) / E(i v)| \text { is a decreasing function of }|v|, 0<\theta<1 \text {. }
$$

Proof of (3.1). Let $\Re(w)>0$, and $N$ be the integer defined by

$$
\left(\rho+N-\frac{1}{2}\right) \cos \phi \leqslant r<\left(\rho+N+\frac{1}{2}\right) \cos \phi .
$$

On considering separately the factors in (2.2) for which $1 \leqslant n<N, n=\mathrm{N}$ and $n \geqslant N+1$, as Levinson does, we get

$$
\begin{aligned}
& |E(w)| \leqslant\left|\frac{\Gamma(\rho+N+1+\delta) \Gamma(\rho+N+1-\delta-w)}{\Gamma(\rho+N+1-\delta) \Gamma(\rho+N+1+\delta-w)}\right| \\
& \quad \cdot\left|\frac{\Gamma(\rho+1-\delta) \Gamma(1-\delta)\left(\lambda_{N}-w\right)}{\Gamma(\rho+1-\delta-w) \Gamma(1-\delta+w)(\rho+N-\delta-w)}\right| .
\end{aligned}
$$

By Stirling's theorem the first factor in (3.9) does not exceed

$$
A_{1}\left(\rho+N-\frac{1}{2}\right)^{2 \delta} /|\rho+N+1-\delta-w|^{2 \delta} ;
$$

while the second factor does not exceed

$$
\frac{A_{2}}{r^{\rho+1-2} \delta}\left|\frac{\left(\lambda_{N}-w\right) \sin \pi(w+\delta-\rho)}{\pi(w+\delta-N-\rho)}\right| .
$$

Now when $|w+\delta-N-\rho|<\frac{1}{2}, \mid \sin \pi(w+\delta-\rho) / \pi(w+\delta-N-\rho)<A_{3}$; and by (2.1), $\left|\lambda_{N}-w\right|<1$. When $|w+\delta-N-\rho| \geqslant \frac{1}{2},|\sin \pi(w+\delta-\rho)| \leqslant$ $A_{4} \exp (\pi|v|)$, and $\left|\left(\lambda_{N}-w\right) /(w+\delta-N-\rho)\right| \leqslant 1+2 \delta \alpha_{N} /|w+\delta-N-\rho|$ $<2$. Thus in all cases the second factor in (3.9) does not exceed

$$
A_{5} \exp (\pi|v|) / r^{\rho+1-2 \delta} \text {. }
$$

We prove now that

$$
b d\{|\rho+N+1-\delta-w| \cos \phi\}>0 .
$$


For by (3.21), (3.8), when $0 \leqslant|\phi| \leqslant \pi / 4$, $|\rho+N+1-\delta-w| \cos \phi \geqslant 2^{-\frac{1}{2}}(\rho+N+1-\delta-u)>$ $2^{-\frac{1}{2}}\left[\left(\rho+N+\frac{1}{2}\right) \sin ^{2} \phi+\frac{1}{2}-\delta\right] \geqslant 2^{-\frac{1}{2}}\left(\frac{1}{2}-\delta\right)$;

and when $\pi / 4<|\phi|<\pi / 2$,

$$
|\rho+N+1-\delta-w| \cos \phi \geqslant(\rho+N+1-\delta-u) /\left(\rho+N+\frac{1}{2}\right)
$$

by $(3.8)$ and $r \geqslant 1$.

$$
\geqslant \sin ^{2} \phi+\left(\frac{1}{2}-\delta\right) /\left(\rho+N+\frac{1}{2}\right) \geqslant \frac{1}{2} \text {, }
$$

From (3.9), (3.10), and (3.12),

$$
\begin{aligned}
|E(w)| & \leqslant A_{6}\left(\rho+N-\frac{1}{2}\right)^{2 \delta} \exp (\pi|v|) / r^{\rho+1-2 \delta}|\rho+N+1-\delta-w|^{2 \delta}, \\
& \leqslant A_{7} r^{2 \delta} \exp (\pi|v|) / r^{\rho+1-2 \delta}\{|\rho+N+1-\delta-w| \cos \phi\}^{2 \delta}, \\
& \leqslant A_{8} \exp (\pi|v|) / r^{\rho+1-4 \delta}
\end{aligned}
$$

by (3.13). This proves (3.1) for $\Re(w)>0$; and the assertion is seen to be true for $\Re(w)<0$ by applying the same argument to $E(-w)$.

Remark on the proof of (3.4). Levinson's method may be used to show that when $\rho+N \leqslant u \leqslant \rho+2 N$,

$$
\begin{aligned}
|E(u)| \leqslant \frac{A_{9} N^{4 \delta-\rho-1}}{(\rho+2 N+1-u)^{2} \delta} \prod_{N}^{2 N}\left|\frac{\rho+n-\delta+\alpha_{n}-u-2 i}{\rho+n-\delta-u-2 i}\right|^{2 \delta}, \\
\quad \int_{\rho+N}^{\rho+2 N}|E(u)|^{q} d u \leqslant A_{10} / N^{q(\rho+1)-1-2 q \delta},
\end{aligned}
$$

provided that

$$
0 \leqslant 2 q \delta<1
$$

If in addition

$$
q(\rho+1-2 \delta)>1,
$$

it follows from (3.15) that $E(u) \in L(0, \infty)$. The final conclusion follows by considering $E(-u)$ in the same way.

It is evident that there always exists a number $q, 1<q \leqslant 2$, satisfying (3.16) and (3.17), for example $q=(1-\Delta)^{-1}$, where $2 \delta<\Delta<1-2 \delta$.

Proof of (3.5). Since

$$
\begin{aligned}
\left|\left(1-\frac{i v}{\rho+n+\delta}\right)\left(1+\frac{i v}{n+\delta}\right)\right| & \leqslant\left|\left(1-\frac{i v}{\lambda_{n}}\right)\left(1+\frac{i v}{\mu_{n}}\right)\right| \\
& \leqslant\left|\left(1-\frac{i v}{\rho+n-\delta}\right)\left(1+\frac{i v}{n-\delta}\right)\right|,
\end{aligned}
$$

it follows that

$$
\begin{aligned}
\left|\frac{\Gamma(\rho+1+\delta) \Gamma(1+\delta)}{\Gamma(\rho+1+\delta-i v) \Gamma(1+\delta+i v)}\right| & \leqslant|E(i v)| \\
& \leqslant\left|\frac{\Gamma(\rho+1-\delta) \Gamma(1-\delta)}{\Gamma(\rho+1-\delta-i v) \Gamma(1-\delta+i v)}\right|,
\end{aligned}
$$

and (3.5) then follows from a classical property (9, p. 259) of the $\Gamma$-function. 
Proof of (3.6). We give details for the case $v>0$. Writing

$$
\phi_{n}=\operatorname{amp}\left\{\left(1-i v \theta / \lambda_{n}\right)\left(1+i v \theta / \mu_{n}\right) /\left(1-i v / \lambda_{n}\right)\left(1+i v / \mu_{n}\right)\right\},
$$

and using the inequalities for $\lambda_{n}, \mu_{n}$ and $\rho$ in (2.1), we have

$$
\begin{aligned}
\phi_{n} & =\arctan \left(\frac{v(1-\theta) \lambda_{n}}{v^{2} \theta+\lambda_{n}^{2}}\right)-\arctan \left(\frac{v(1-\theta) \mu_{n}}{v^{2} \theta+\mu_{n}^{2}}\right), \\
& <\arctan \left(\frac{v(1-\theta)(\rho+n+\delta)}{v^{2} \theta+(\rho+n-\delta)^{2}}\right)-\arctan \left(\frac{v(1-\theta)(n-\delta)}{v^{2} \theta+(n+\delta)^{2}}\right), \\
& <\arctan \left(\frac{v(1-\theta)(n+1-\delta)}{v^{2} \theta+(n-\delta)^{2}}\right)-\arctan \left(\frac{v(1-\theta)(n-\delta)}{v^{2} \theta+(n+\delta)^{2}}\right), \\
& =\arctan \frac{v(1-\theta)\left\{v^{2} \theta+n^{2}(1+4 \delta)+2 n \delta(1-2 \delta)+\delta^{2}\right\}}{\left[v^{2} \theta+(n-\delta)^{2}\right]\left[v^{2} \theta+(n+\delta)^{2}\right]+v^{2}(1-\theta)^{2}(n+1-\delta)(n-\delta)} .
\end{aligned}
$$

On observing that

$$
0<v^{2} \theta+n^{2}(1+4 \delta)+2 n \delta(1-2 \delta)+\delta^{2}<2\left[v^{2} \theta+(n+\delta)^{2}\right],
$$

we see that $\phi_{n}<\arctan \left\{2 v(1-\theta) /\left[v^{2} \theta+(n-\delta)^{2}\right]\right\}$. A similar argument applied to $-\phi_{n}$ gives

and thus

$$
-\phi_{n}<\arctan \left\{3 v(1-\theta) /\left[v^{2} \theta+(n-\delta)^{2}\right]\right\} ;
$$

$$
\left|\phi_{n}\right|<\arctan \left\{3 v(1-\theta) /\left[v^{2} \theta+(n-\delta)^{2}\right]\right\} .
$$

It then follows easily that $|\operatorname{amp}[E(i v \theta) / E(i v)]|<A(1-\theta)$, the constant being independent of $v$.

Proof of (3.7). Let $\Lambda$ be the region consisting of the $w$-plane from which the points $v=0,|u| \geqslant 1-\delta$ have been removed. Then the series

$$
\sum_{1}^{\infty}\left(\frac{w}{\lambda_{n}-w}-\frac{\theta w}{\lambda_{n}-\theta w}\right), \quad \sum_{1}^{\infty}\left(\frac{w}{\mu_{n}-w}-\frac{\theta w}{\mu_{n}-\theta w}\right)
$$

converge absolutely and uniformly in any compact subset of $\Lambda$, and

$$
\begin{aligned}
\frac{d}{d v} \log \left[\frac{E(i v \theta)}{E(i v)}\right]=i \sum_{1}^{\infty}\left(\frac{\lambda_{n}+i v}{\lambda_{n}^{2}+v^{2}}-\frac{\theta\left(\lambda_{n}+i v \theta\right)}{\lambda_{n}^{2}+v^{2} \theta^{2}}\right) \\
-i \sum_{1}^{\infty}\left(\frac{\mu_{n}-i v}{\mu_{n}^{2}+v^{2}}-\frac{\theta\left(\mu_{n}-i v \theta\right)}{\mu_{n}^{2}+v^{2} \theta^{2}}\right), \\
\Re\left\{\frac{d}{d v} \log \frac{E(i v \theta)}{E(i v)}\right\}=-\sum_{1}^{\infty}\left[\frac{v \lambda_{n}^{2}\left(1-\theta^{2}\right)}{\left(\lambda_{n}^{2}+v^{2}\right)\left(\lambda_{n}^{2}+v^{2} \theta^{2}\right)}+\frac{v \mu_{n}^{2}\left(1-\theta^{2}\right)}{\left(\mu_{n}^{2}+v^{2}\right)\left(\mu_{n}^{2}+v^{2} \theta^{2}\right)}\right]<0 .
\end{aligned}
$$

It follows that $|E(i v \theta) / E(i v)|$ is a decreasing function of $|v|$.

4. Representation of the operator. Let $p$ be the index conjugate to $q$, so that $p \geqslant 2$. By (3.4), the function

$$
k(y)=\lim _{R \rightarrow \infty}^{(p)}(2 \pi)^{-\frac{1}{2}} \int_{-R}^{R} E(u) \exp (-i u y) d u
$$


exists and belongs to $L^{p}(-\infty, \infty)$. On considering the contour integral $\int E(w) \exp (-i y w) d w$ taken round the boundary of the semi-circular disc $|w| \leqslant R, v \geqslant 0$, we see that the part of the integral taken round the arc of the semi-circle is $O\left(R^{-\beta}\right),(R \rightarrow \infty)$ when $|y|>\pi$, where $\beta=\rho+1-4 \delta>0$ in (3.1). Thus

$$
k(y)=0
$$

$p . p$. in $|y|>\pi$.

Since $E(u)$ is continuous

$$
\begin{aligned}
E(u) & =\lim _{R \rightarrow \infty}(2 \pi)^{-\frac{1}{2}} \int_{-R}^{R} k(y)[1-|y| / R] \exp (\text { iuy }) d y, \\
& =(2 \pi)^{-\frac{1}{2}} \int_{-\pi}^{\pi} k(y) \exp (\text { iuy }) d y,
\end{aligned}
$$

and $k(y) \in L^{2}(-\pi, \pi)$ by $(4.1)$ and $p \geqslant 2$.

It is easily seen that for complex $w$, the integral

$$
(2 \pi)^{-\frac{1}{2}} \int_{-\pi}^{\pi} k(y) \exp (i y w) d y
$$

defines an entire function, and by (4.2) we may write

$$
E(w)=(2 \pi)^{-\frac{1}{2}} \int_{-\pi}^{\pi} k(y) \exp (i y w) d y .
$$

In proving the inversion theorem we shall make use of the function $E(\theta w)$, $0<\theta \leqslant 1$, for which we prove

$$
\left|E\left(\theta r_{n}\right)\right| \leqslant A(1-\theta) r_{n}
$$

where $r_{n}$ stands for $\lambda_{n}$ or $-\mu_{n}$ and the constant is independent of $n$.

Let $m(y, \alpha)=\exp (-i y \alpha \theta)-\exp (-i y \alpha)$. Then for $|y|<\pi$, and $0<\theta \leqslant 1$,

$$
\begin{gathered}
|m(y, \alpha)|=|\alpha|\left|\int_{\theta y}^{y} \exp (-i t \alpha) d t\right| \leqslant \pi|\alpha|(1-\theta) ; \\
\left|E\left(-\theta \mu_{k}\right)-E\left(-\mu_{k}\right)\right|=(2 \pi)^{-\frac{1}{2}}\left|\int_{-\pi}^{\pi} k(y) m\left(y, \mu_{k}\right) d y\right| \leqslant A(1-\theta) \mu_{k},
\end{gathered}
$$

by the Schwarz inequality.

With the usual interpretation of $\exp (a D)$ as a shift operator, we have formally

$$
\begin{aligned}
D E(D) . f(x) & =\lim _{\theta \rightarrow 1} D E(\theta D) \cdot f(x), \\
& =\lim _{\theta \rightarrow 1}(2 \pi)^{-\frac{1}{2}} \int_{-\pi}^{\pi} k(y) \exp (i y \theta D) d y \cdot f^{\prime}(x), \\
& =\lim _{\theta \rightarrow 1}(2 \pi)^{-\frac{1}{2}} \int_{-\pi}^{\pi} k(y) f^{\prime}(x+i y \theta) d y .
\end{aligned}
$$

We therefore define the operation $D E(D) . f(x)$ by

$$
D E(D) . f(x)=\lim _{\theta \rightarrow 1}(2 \pi)^{-\frac{1}{2}} \int_{-\pi}^{\pi} k(y) f^{\prime}(x+i y \theta) d y .
$$

5. Properties of the nucleus. Denoting the strip $|y|<\pi$ of the $z$-plane by $B$, we prove the following propositions: 
(5.1) the integral (2.3) defining $G(z)$ converges absolutely when $z \in B$, converges uniformly when $z$ belongs to a compact subset of $B$, and therefore defines a function analytic in $B$;

$$
\begin{aligned}
& G(z)=\left\{\begin{array}{lr}
1+O\left[\exp \left(-x \mu_{1}\right)\right], & (x \rightarrow \infty \text { in } B), \\
O\left[\exp \left(x \lambda_{1}\right)\right], & (x \rightarrow-\infty \text { in } B) ;
\end{array}\right.
\end{aligned}
$$

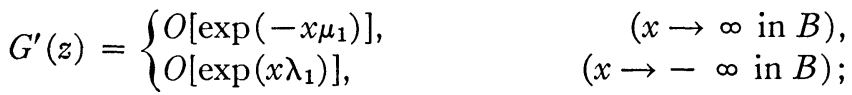

(5.4) when $z_{0} \in B$, there is a constant $R_{0}$ such that the integrals

$$
\int_{-\infty}^{-R_{0}}\left|\frac{d}{d t} \frac{G(z-t)}{G\left(z_{0}-t\right)}\right| d t, \quad \int_{R_{0}}^{\infty}\left|\frac{d}{d t} \frac{G(z-t)}{G\left(z_{0}-t\right)}\right| d t
$$

converge uniformly when $z$ belongs to any compact subset of $B$.

Proof of (5.1). By (2.3), (3.2),

$\left|\frac{1}{2 \pi i} \int_{c-i \infty}^{c+i \infty} \frac{\exp (z w) d w}{w E(w)}\right| \leqslant A_{1} \exp (c x) \int_{-\infty}^{\infty} \exp [-y v-(\pi-\epsilon)|v|] d v$

since $|y|<\pi$ in $B$. This inequality is sufficient to establish the assertions of (5.1).

Proof of (5.2), (5.3). On account of the classical properties of Dirichlet series it is sufficient to show that

$$
G(z)=\left\{\begin{aligned}
1-\sum_{1}^{\infty} \exp \left(-z \mu_{n}\right) / \mu_{n} E^{\prime}\left(-\mu_{n}\right), & (x>0,|y|<\pi), \\
-\sum_{1}^{\infty} \exp \left(z \lambda_{n}\right) / \lambda_{n} E^{\prime}\left(\lambda_{n}\right), & (x<0,|y|<\pi),
\end{aligned}\right.
$$

the Dirichlet series converging absolutely in the indicated regions.

Details are given for the case $x<0$. Designating the points $c-i R$, $c+R \cot \beta-i R, c+R \cot \beta+i R$ and $c+i R$ by $A, B, C$ and $D$ respectively, where $0<\beta<\frac{1}{2} \pi$, let $L$ be the contour formed by the linear segments $A B$ and $C D$ and the circular arc $|w-c|=R$ joining to $B$ to $C$. Consider

$$
I=\int_{L} \exp (z w) d w / w E(w)
$$

By using (3.2) and $x<0,0<\beta<\frac{1}{2} \pi,|y|<\pi$, and estimating the integrals along $A B, B C$ and $C D$ separately, we see that $I=o(1)$ as $R \rightarrow \infty$. The second equation in (5.5) then follows from the definition of $G(z)$ and the calculus of residues.

Proof of (5.4). When $x \neq 0, G(z)$ is represented by the absolutely convergent Dirichlet series (5.5), and it is well known that functions so defined can have but a finite number of zeros. Since $z_{0}$ is given, and $X_{1} \leqslant x \leqslant X_{2}$ 
in the compact subset of $B$, we may choose $R_{0}$ so that $G\left(z_{0}-t\right)$ does not vanish for $|t| \geqslant R$. It then follows easily that

$$
\left|\frac{d}{d t} \frac{G(z-t)}{G\left(z_{0}-t\right)}\right|=\left\{\begin{array}{lr}
O\left[\exp \left(t \mu_{1}\right)\right] & (t \rightarrow-\infty), \\
O\left\{\exp \left[-t\left(\lambda_{2}-\lambda_{1}\right)\right]\right\} & (t \rightarrow \infty),
\end{array}\right.
$$

where the constants are independent of $z$. These estimates are sufficient for the proof.

6. Properties of the transform. The following theorem gives properties of the functions $f(x)$ and $\phi(t)$ in (1.1) which will be used later.

THEOREM I. Let $\phi(t) \in L(0, R)$ for any $R$ and be such that the integral (1.1) converges for at least one $z$ in $B$, and let $\Phi(t)=\int_{0}^{t} \phi(u) d u$ : then

(6.1) the integral (1.1) converges for all $z$ in $B$, and defines a function analytic in $B$;

for any positive $\Delta$.

$$
\Phi(t)=\left\{\begin{array}{lr}
o\left(\exp t \lambda_{1}\right) & (t \rightarrow \infty), \\
o(\exp -t \Delta) & (t \rightarrow-\infty),
\end{array}\right.
$$

Proof of (6.1). On account of (5.4), the method of Widder-Hirschmann may be used (4, pp. 691-692).

Proof of (6.2). It follows from the representation (5.5) of $G(z)$ for $x \neq 0$ that $G(z)$ and $G^{\prime}(z)$ have at most a finite number of zeros. Let $A$ be a positive number such that neither $G(z)$ nor $G^{\prime}(z)$ vanishes for $|x| \geqslant A$. To prove the first assertion, define $\psi(t)=\int_{0}^{t} G(-A-u) d \Phi(u)$. Then by hypothesis, $\psi(\infty)$ is finite, and

$$
\begin{aligned}
\Phi(t) \exp \left(-t \lambda_{1}\right) & =\exp \left(-t \lambda_{1}\right) \int_{0}^{t} d \psi(u) / G(-A-u) \\
& =\exp \left(-t \lambda_{1}\right)\left[\frac{\psi(t)}{G(-A-t)}-\int_{0}^{t} \frac{\psi(u) G^{\prime}(-A-u) d u}{G^{2}(-A-u)}\right], \\
& =o(1)
\end{aligned}
$$

as $t \rightarrow \infty$ by l'Hopital's rule, and (5.5).

To prove the second assertion, write $\psi(t)=\int_{0}^{t} G(A-u) d \Phi(u)$; and in the same way, $\Phi(t) \exp (t \Delta)=o(1),(t \rightarrow-\infty)$.

It is convenient at this point to establish some properties of the function

$$
K(x, \theta)=\theta(2 \pi)^{-\frac{1}{2}} \int_{-\pi}^{\pi} k(y) G^{\prime}(x+i y \theta) d y .
$$

These properties are:

$$
K(x, \theta)=\left\{\begin{array}{lr}
(1-\theta) O\left[\exp \left(-x \mu_{1}\right),\right. & (x \rightarrow \infty), \\
(1-\theta) O\left[\exp \left(x \lambda_{1}\right)\right], & (x \rightarrow-\infty),
\end{array}\right.
$$

with similar estimates for $K^{\prime}(x, \theta)$;

$$
K(x, \theta)=O\left[(1-\theta)^{-1}\right] \text { uniformly in } x \text { as } \theta \rightarrow 1 \text {; }
$$

(6.6) when $x$ is positive, $\lim _{\theta \rightarrow 1} \int_{0}^{x} K(t, \theta) d t=\frac{1}{2}=\lim _{\theta \rightarrow 1} \int_{-x}^{0} K(t, \theta) d t$. 
Proof of (6.4). Details are given for the case $x>0$.

$$
\begin{array}{rlrl}
|K(x, \theta)| & =\left|\theta(2 \pi)^{-\frac{1}{2}} \int_{-\pi}^{\pi} k(y) d y(2 \pi i)^{-1} \int_{c-i_{\infty}}^{c+i_{\infty}} \exp [w(x+i y \theta)] d w / E(w)\right| \\
& =\left|(2 \pi i)^{-1} \int_{c-i_{\infty}}^{c+i_{\infty}} E(\theta w) \exp (x w) d w / E(w)\right|, & \\
& =\left|\sum_{1}^{\infty} E\left(-\theta \mu_{n}\right) \exp \left(-x \mu_{n}\right) / E^{\prime}\left(-\mu_{n}\right)\right|, & \\
& \leqslant A_{1}(1-\theta) \sum_{1}^{\infty} \mu_{n} \exp \left(-x \mu_{n}\right) /\left|E^{\prime}\left(-\mu_{n}\right)\right|, & \text { by (4.4) }
\end{array}
$$

and as $x>0$ and (3.3) guarantee the convergence of this series, our assertion is proved.

Proof of (6.5). By (4.3), (6.3) and Cauchy's theorem,

$$
\begin{aligned}
K(x, \theta) & =\theta(2 \pi)^{-\frac{1}{2}} \int_{-\pi}^{\pi} k(y) d y(2 \pi i)^{-1} \int_{-i_{\infty}}^{i_{\infty}} \exp [w(x+i y \theta)] d w / E(w), \\
& =\theta(2 \pi)^{-1} \int_{-\infty}^{\infty} \exp (i x v) E(i v \theta) d v / E(i v) .
\end{aligned}
$$

Using the fact that for $0<\theta<1$,

$$
\left|\left(1-i v \theta / \lambda_{n}\right) /\left(1-i v \lambda_{n}\right)\right| \text { and }\left|\left(1+i v \theta / \mu_{n}\right) /\left(1+i v / \mu_{n}\right)\right|
$$

are less than unity, we have

$$
\left|\frac{1-i v \theta /(\rho+n-\delta)}{1-i v /(\rho+n-\delta)}\right| \leqslant\left|\frac{1-i v \theta / \lambda_{n}}{1-i v / \lambda_{n}}\right| \leqslant\left|\frac{1-i v \theta /(\rho+n+\delta}{1-i v /(\rho+n+\delta}\right|,
$$

with similar inequalities involving $\mu_{n}$. Hence

$$
\begin{aligned}
\left|\frac{\Gamma(\rho+1-\delta-i v)}{\Gamma(1-\delta+i v)}\right| \leqslant\left|\frac{E(i v \theta)}{E(i v)}\right| & \\
& \leqslant\left|\frac{\Gamma(\rho+1+\delta-i v) \Gamma(1+\delta+i v)}{\Gamma(\rho+1+\delta-i v \theta) \Gamma(1+\delta+i v \theta)}\right|,
\end{aligned}
$$

and by (9, p. 259),

$$
|E(i v \theta) / E(i v)| \sim A \exp [-\pi|v|(1-\theta)] \quad(|v| \rightarrow \infty) .
$$

Since $0<\theta<1$, this is sufficient to prove our result.

Proof of (6.6). Write $I=\int_{0}^{x} K(t, \theta) d t$, where $x \neq 0$. Then

$$
\begin{aligned}
I & =\theta(2 \pi)^{-\frac{1}{2}} \int_{0}^{x} d t \int_{-\pi}^{\pi} k(y) G^{\prime}(t+i y \theta) d y, \\
& =\theta(2 \pi)^{-\frac{1}{2}} \int_{-\pi}^{\pi} k(y)[G(x+i y \theta)-G(i y \theta)] d y,
\end{aligned}
$$

the interchange of the integrations being justified, since $k(y) \in L^{2}(-\pi, \pi)$ and 
Thus

$$
\begin{aligned}
\left|G^{\prime}(t+i y \theta)\right| & =\left|(2 \pi i)^{-1} \int_{c-i_{\infty}}^{c+i_{\infty}} \exp [w(t+i y \theta)] d w / E(w)\right|, \\
& =\left|(2 \pi)^{-1} \int_{-\infty}^{\infty} \exp (-v y \theta+i v t) d v / E(i v)\right|, \\
& =\int_{-\infty}^{\infty} \exp [-\pi|v|(1-\theta)] O\left[|v|^{\rho+1+2 \delta}\right] d v, b y(3.5), \\
& =O\left[(1-\theta)^{-\rho-2-2 \delta}\right] .
\end{aligned}
$$

$I=\theta(2 \pi)^{-\frac{1}{2}} \int_{-\pi}^{\pi} k(y) d y(2 \pi i)^{-1} \int_{c-i_{\infty}}^{c+i_{\infty}}\{\exp [w(x+i y \theta)]-\exp (i y \theta w)\} d w / w E(w)$.

Again by (4.3) and the absolute convergence of the inner integral for $|y|<\pi$, we may interchange the integrations, and get

$$
I=\frac{\theta}{2 \pi} \int_{-\infty}^{\infty} \frac{[\exp (i x v)-1] E(i v \theta)}{i v E(i v)} d v,
$$

the application of Cauchy's theorem being justified by the analyticity of $[\exp (x w)-1] / w$ at the origin.

We observe next that $E(\theta w) / E(w)$ is real when $w$ is real. Hence $I=P-Q$, where

$$
\begin{aligned}
& Q=\frac{\theta}{2 \pi} \int_{0}^{\infty} \frac{1-\cos x v}{v} \Im\left[\frac{E(i v \theta)}{E(i v)}\right] d v, \\
& P=\frac{\theta}{2 \pi} \int_{0}^{\infty} \frac{\sin x v}{v} \Re\left[\frac{E(i v \theta)}{E(i v)}\right] d v .
\end{aligned}
$$

It is then sufficient to show that

$$
\begin{array}{ll}
Q \rightarrow 0, & \theta \rightarrow 1, \\
P \rightarrow \frac{1}{2}, & \theta \rightarrow 1 .
\end{array}
$$

To prove (6.9), it is sufficient to consider

We then have

$$
Q_{1}=\int_{1}^{\infty} \frac{1-\cos x v}{v} \Im\left[\frac{E(i v \theta)}{E(i v)}\right] d v .
$$

$$
\begin{aligned}
\left|Q_{1}\right| & =\left|\int_{1}^{\infty} \frac{1-\cos x v}{v}\right| \frac{E(i v \theta)}{E(i v)}\left|\sin \operatorname{amp}\left[\frac{E(i v \theta)}{E(i v)}\right] d v\right|, \\
& \leqslant A_{1}(1-\theta) \int_{1}^{\infty} v^{-1} \exp [-\pi v(1-\theta)] d v, \quad \text { by (3.6) and (6.7), } \\
& =A_{1}(1-\theta) \int_{\pi(1-\theta)}^{\infty} t^{-1} \exp (-t) d t<A_{2}(1-\theta)^{\frac{1}{2}} \Gamma\left(\frac{1}{2}\right) .
\end{aligned}
$$

Thus $Q_{1}$, and consequently $Q$ tends to zero as $\theta$ tends to unity.

To prove (6.10), we observe that on account of (3.7),

$$
\int_{0}^{\infty} \frac{\sin x v}{v}\left|\frac{E(i v \theta)}{E(i v)}\right| d v
$$

converges uniformly in $\frac{1}{2} \leqslant \theta \leqslant 1$; and from (3.6), that $\Re[E(i v \theta) / E(i v)]$ is 
positive when $\theta$ is close to unity. It is therefore sufficient to prove that $C(\theta) \rightarrow 0(\theta \rightarrow 1)$, where

But

$$
C(\theta)=\int_{0}^{\infty} \frac{\sin x v}{v}\left\{\left|\frac{E(i v \theta)}{E(i v)}\right|-\Re\left[\frac{E(i v \theta)}{E(i v)}\right]\right\} d v .
$$

$$
\begin{aligned}
|C(\theta)| & \leqslant \int_{0}^{\infty}\left|\frac{E(i v \theta)}{E(i v)}\right|\left\{1-\cos \operatorname{amp}\left[\frac{E(i v \theta)}{E(i v)}\right]\right\} d v, \\
& \leqslant A_{3}(1-\theta)^{2} \int_{0}^{\infty} \exp [-\pi v(1-\theta)] d v, \\
& =O[(1-\theta)],
\end{aligned}
$$

7. The inversion theorems. The main result is

THEOREM II. Let $\phi(t) \in L(0, R)$ for any $R$ and be such that the integral (1.1) converges for at least one $z$ in the strip $B:$ then if $f(z)$ is defined by (1.1) and $D E(D) . f(x)$ by $(4.5)$,

$$
D E(D) \cdot f(x)=\frac{1}{2}[\phi(x+)+\phi(x-)],
$$

whenever the right-hand side has a meaning.

For

$$
\begin{aligned}
D E(D) . f(x) & =\lim _{\theta \rightarrow 1}(2 \pi)^{-\frac{1}{2}} \int_{-\pi}^{\pi} k(y) d y \int_{-\infty}^{\infty} G^{\prime}(x-t+i y \theta) \phi(t) d t, \\
& =\lim _{\theta \rightarrow 1} \int_{-\infty}^{\infty} \phi(t) d t(2 \pi)^{-\frac{1}{2}} \int_{-\pi}^{\pi} k(y) G^{\prime}(x-t+i y \theta) d y, \\
& =\lim _{\theta \rightarrow 1} \int_{-\infty}^{\infty} K(x-t, \theta) \phi(t) d t,
\end{aligned}
$$

the interchange of the integrations being justified by the uniform convergence of (1.1) in any compact subset of $B$, and the fact that $k(y) \in L^{2}(-\pi, \pi)$. It is sufficient to prove

$$
\begin{aligned}
& \int_{-\infty}^{x} K(x-t, \theta) \phi(t) d t \rightarrow \frac{1}{2} \phi(x-), \\
& \int_{x}^{\infty} K(x-t, \theta) \phi(t) d t \rightarrow \frac{1}{2} \phi(x+),
\end{aligned}
$$

as $\theta \rightarrow 1$.

We give details for (7.1). Let $T>0$, and write

$$
\int_{x-T}^{x} K(x-t, \theta)[\phi(t)-\phi(x-)] d t=\int_{0}^{T} K(t, \theta)[\phi(x-t)-\phi(x-)] d t=J(0, T) .
$$

Then

$$
\begin{aligned}
|J[0, \pi(1-\theta)]| & \leqslant \int_{0}^{\pi(1-\theta)}|\phi(x-t)-\phi(x-)||K(t, \theta)| d t \\
& \leqslant A(1-\theta)^{-1} \int_{0}^{\pi(1-\theta)}|\phi(x-t)-\phi(x-)| d t \\
& =o(1)
\end{aligned}
$$


as $\theta \rightarrow 1$, by (6.5). Next by (6.4),

$$
\begin{aligned}
|J[\pi(1-\theta), T]| & \leqslant A(1-\theta) \int_{\pi(1-\theta)}^{T}|\phi(x-t)-\phi(x-)| \exp \left(-t \mu_{1}\right) d t \\
& =O(1-\theta) .
\end{aligned}
$$

Thus by (6.6), (7.3) and (7.4)

$$
\lim _{\theta \rightarrow 1} \int_{x-T}^{x} K(x-t, \theta) \phi(t) d t=\frac{1}{2} \phi(x-) .
$$

It remains to prove that $\int_{-\infty}^{x-T} K(x-t, \theta) \phi(t) d t \rightarrow 0$ as $\theta \rightarrow 1$. As this integral need not converge absolutely, we write it as

$$
\left[K^{\prime}(x-t, \theta) \Phi(t)\right]_{-\infty}^{T}+\int_{-\infty}^{x-T} K^{\prime}(x-t, \theta) \Phi(t) d t .
$$

By (6.2) and (6.4) the integrated term $=o(1)$; and for the same reason

$$
\left|\int_{-\infty}^{x-T} K^{\prime}(x-t, \theta) \Phi(t) d t\right|=\left|\int_{T}^{\infty} K^{\prime}(t, \theta) \Phi(x-t) d t\right|=O(1-\theta) .
$$

Since (7.2) may be proved in the same way, the theorem is complete.

The proof of the following theorem is similar:

THEOREM III. Let $f(z)=\int_{-\infty}^{\infty} G(z-t) d \alpha(t)$, where $\alpha(t)$ is a normalized function of bounded variation in any finite interval: then if this integral converges for any $z$ in $B$, it converges for all such $z$, converges uniformly in any compact subset of $B$, and defines a function analytic in $B$. Also

$$
\lim _{\theta \rightarrow 1}(2 \pi)^{-\frac{1}{2}} \int_{x_{1}}^{x_{2}} d x \int_{-\pi}^{\pi} k(y) f^{\prime}(x+i y \theta) d y=\alpha\left(x_{2}\right)-\alpha\left(x_{1}\right) .
$$

8. Remarks. In the proof of (5.4) we have used the fact that from its representation (5.5) as a Dirichlet series, the nucleus $G(z)$ has but a finite number of zeros. Hirschmann and Widder (8, p. 159) have shown that a more general nucleus has no zeros on the real axis, and it is certainly true that $G(i y) \neq 0$ for $|y|<\pi$. The proof that $G(z)$ does not vanish in $B$ seems to be connected with properties of functions defined by Dirichlet series with coefficients of alternating sign, and will be dealt with elsewhere.

\section{REFERENCES}

1. N. Wiener, The operational calculus, Math. Ann., 95 (1925-26), 557-584.

2. D. V. Widder, Inversion formulas for convolution transforms, Duke Math. J., 14 (1947), 217-249.

3. - - The Stieltjes transform, Trans. Amer. Math. Soc., 43 (1938), 7-60.

4. I. I. Hirschmann, Jr. and D. V. Widder, Generalized inversion formulas for convolution transforms, Duke Math. J., 15 (1948), 659-696.

5. D. B. Sumner, An inversion formula for the generalized Stieltjes transform, Bull. Amer. Math. Soc., 55 (1949), 174-183. 
6. I. I. Hirschmann, Jr. and D. V. Widder, Convolution transforms with complex kernels, Pacific J. Math., 1 (1951), 211-225.

7. N. Levinson, Gap and density theorems (Amer. Math. Soc. Coll., vol. XXVI, 1940).

8. I. I. Hirschmann, Jr. and D. V. Widder, Inversion of a class of convolution transforms, Trans. Amer. Math. Soc., 66 (1949), 135-201.

9. E. C. Titchmarsh, The theory of functions (1st. ed., Oxford, 1932).

10. G. H. Hardy and N. Levinson, Inequalities satisfied by a certain definite integral, Bull. Amer. Math. Soc., 43 (1937), 709-716.

Hamilton College, McMaster University, and

Research Institute of the Canadian Mathematical Congress. 